\title{
Study on the Subject Collaborative Interaction Model of the Knowledge Sharing of Industry-University Cooperative
}

\author{
Ningning $\operatorname{Yan}^{1, a^{*}}$ \\ ${ }^{1}$ School of Business Administration, Jing De Zhen Ceramic Institute, Jiangxi Province, China \\ asemail2008@163.com
}

\begin{abstract}
Keywords: Industry-University cooperative; Knowledge sharing; Collaborative interaction; Subject collaborative; Collaborative interaction model.
\end{abstract}

\begin{abstract}
Knowledge assets are the sum of the knowledge organization has which can bring competitive advantage and benefit, including patent technology, technology secret, management experience, learning ability and so on. The complexity and cross disciplinary characteristics of technological innovation makes the knowledge assets of single organization can't meet the requirements of innovation. At this time, the organization needs to seek the necessary knowledge assets from outside to make up for the deficiency of the organization's knowledge assets. In the process of cooperative innovation knowledge sharing, compared with the individual innovation and market transactions, it will produce the synergistic effect that both do not have, namely the revenue of multiple subjects cooperation greater than the sum of independent innovation achieve gains. This paper analyzes the subject collaborative interaction concept and role of industry-university cooperative knowledge sharing, to explore the industry-university cooperative knowledge sharing process, focusing on hybrid collaborative interaction model of industry-university cooperative knowledge sharing based on the role-playing. The research shows that the realization of is greatly promoted the knowledge communication and sharing between the two sides.
\end{abstract}

\section{Introduction}

The collaborative interaction behavior among the subjects is an important manifestation of the subjectivity of the society, is the main difference in the important attribute of traditional organization system, rational, perfect collaborative interaction mechanism is the coordination, cooperation and negotiation among multi agents; it is to reflect the premise of organizations in each of the main role relationship between. Here, it is necessary to make a further description of the concept of collaborative interaction [1]. We analyze the spatial and temporal dimensions of social synergy, and divide social synergy into two patterns: horizontal and vertical. Horizontal coordination phenomenon is one of the oldest and most simple coordination schemes, which refers to the cooperative behavior of multiple individuals to complete a project together. Through this collaboration, people can complete a person unable to complete the large-scale engineering, can also be a segmentation to complete a project in each section, and thus to simplify the complexity of the work, and increase the work efficiency [2]. Vertical coordination is the result of a study or project that can assist or actually contribute to a subsequent project. From the way of social coordination, social synergy is divided into two modes; one is one way and two ways. One way collaborative phenomenon is a cooperative activity that is only for the purpose of a party. One-way collaboration has synergy and the two parts of the collaboration, collaborative planning and coordination of the beneficiaries, is the implementation of collaborative relative to the party. Bilateral collaboration is the synergy of the participants' part or all of their status. This is one of the most common human ways, and it is the form of knowledge sharing. 


\section{The Subject Collaborative Interaction Concept and Function of Industry-University Cooperative Knowledge Sharing}

The association points out that there is a parameter that determines the degree of order of the system in the course of the interaction. It is also the main aspect of the main contradiction and the contradiction of the state of things. This parameter will be Hawking called "order parameter". At the same time, Hawkins's research also pointed out that the order parameter is the system evolution speed is the slowest of the subsystem, that is slow variables determine the evolution process of the system, determine the evolution results in the appearance of the structure and function of. In the system, the fast variable is subordinate to the slow variable, and the fast variable is controlled by the slow variable. "Order parameter" has prompted the combination of different elements together to evolve and develop, and lead the system towards a more advanced and orderly structure. For the knowledge sharing of the industry cooperation, collaborative interaction is the interaction between the enterprise knowledge subject and the university knowledge subject, which forms the order parameter, which determines the main development direction of the industry cooperation. This kind of cooperative interaction is the result of the integration of the complementary knowledge resources between enterprises and universities, so that it has the advantages of $1+1>2$ [3].

First, strictly speaking, among the subject and the object of knowledge interaction behavior is still the main body and the external environment are the role of a way, but because of its special interactive objects and its significance. Therefore, the individual research is very necessary.

Secondly, we from the angle of subject organization, interactive collaborative subject their closely related to the corresponding roles and goals. Therefore, strictly speaking, collaborative interaction objects involved in an interactive process is to appear in the role of identity. In particular, the interaction between the agents and the interaction strategy, in fact, is the interaction between the main body of the agreement and interaction strategy. Also, here the interaction is a broad concept; it refers to the subject knowledge shared form of communicative action, so that production and study cooperation knowledge sharing between the subject and the object of any communication, cooperation, negotiation or arbitration belong to this category [4].

The interaction of the subject is the intrinsic factor of the interaction behavior of the subject. In the light of human social organization, we will find that the interaction of any rational entity must be related to the purpose, object, and mode and so on. Therefore, as the abstract of the rational entity, the study of the interaction of the subject should also be solved from how to solve these three problems, namely: first, why the interaction? Secondly, and who interact? Thirdly, how to interact? The first problem and the second problem involves the interaction of the main motivation of the generation and interaction in the process of reasoning, we will go through the analysis of production and learning cooperation knowledge sharing main body mental state changes are explained; the third problem relates to the realization of the interactive, we will in the analysis of the cooperation knowledge sharing subject interactive hierarchy model was put forward based on the factor structure of the, and through the analysis of the model, to produce learn collaboration of knowledge sharing subject interaction mechanism framework [5].

\section{The Subject Collaborative Interaction Process of Industry-University Cooperative Knowledge Sharing}

The knowledge sharing of production and learning cooperation is done by different subjects. Any entities in the real environment, whose function are always determined by its inherent structural characteristics. The inner structure of the subject is closely related to its functional characteristics. The different environment, different application purpose and the concrete realization way are difference; all makes the main body in many aspects to show the difference. The two different types of enterprises, which are the main body of the enterprise and the University, form a cooperative relationship, which is the process of producing new knowledge [6]. 
Cooperative interaction of knowledge sharing in industry is often based on a project, through collaborative interaction can generate new knowledge and make it quickly into the enterprise technical ability and the University's scientific research and personnel training advantages. From the framework of this model, we can see that the cooperative interaction of the knowledge sharing in the production process can be divided into the following three stages.

First stage: enterprise or university from their own needs (such as the development of new products, staff training, the reform of the production process or scientific research, talent cultivation and social service of, put forward corresponding research topic, enterprise and University on the subject is common knowledge and the technical innovation, the formation of the industry University cooperation of knowledge sharing common vision, at this time, enterprises and universities were reciprocal exchange of knowledge value, establish appropriate cooperation sharing, contract, establish collaborative and interactive platform, gathers the advantage of knowledge resources companies and universities.

The second stage: enterprise and university based on identified in the first phase of shared vision, the two sides in own knowledge selection and overflow, the integration of knowledge and shared knowledge formation, a total of knowledge absorption and application in the process of discovering problems and solving problems, and ultimately collaborative interactive spiral. This includes both the organization of collaborative learning, team learning and knowledge interaction. The each member to participate in the process of the accumulation of knowledge and technology; second, corporate and university staff through close communication, in the knowledge sharing process can be obtained from each other with different characteristics of knowledge; thirdly, knowledge sharing process employees to get the information of the other party processed and integrated, and ultimately achieve the knowledge from the enterprise to the university (university to enterprise) two-way flow, for the realization of the synergistic interaction lay the foundation.

The third stage: in this stage through the cooperation between enterprises and universities, so that the knowledge sharing of the cooperation to create a new value of knowledge. These innovations include not only the creation of tangible resources, but also the promotion of core innovation capability. And the enterprise and the university each ability promotion also needs the enterprise and the university knowledge sharing carrier and the environment guarantee.

It should be pointed out that the completion of this collaborative interaction, enterprises and universities have accumulated the experience of knowledge sharing of production and learning cooperation, in order to build a new partnership for the future, a new round of collaborative interaction laid the foundation.

\section{Hybrid Collaborative Interaction Model of Industry-University Cooperative Knowledge Sharing Based on the Role-Playing}

The knowledge sharing is a complex subject structure. In the process of realizing the value creation, the enterprise and the university are actually playing a role in order to accomplish the task of organization and realize the organization goal. Therefore, in the analysis of production and learning cooperation knowledge sharing coordination, in order to reflect the behavior of the subjects of the reasoning process, we can the idea of integration, build a specific role of compound based on production cooperation study, knowledge sharing main body structure.

Based on role play the main structure of the framework is given for the main body of the main structure of the general description, in order to further reflect the behavior of the subject is proposed in this paper based on the role played by the compound production cooperation study, knowledge sharing and cooperative interaction model.

First of all, we analyze the process of knowledge sharing. In front of us, we divide the factors of knowledge sharing into three processes, namely, self-knowledge selection and spillover, knowledge integration and common knowledge formation, the absorption and application of common knowledge. (1) Its own knowledge selection and overflow. To the organization of its own intellectual screening, 
first in the organization after the collective knowledge express and aggregated combinations of list, identification, classification, refining, etc., if you belong to the core knowledge, the knowledge cannot used to share, still retains in the knowledge base of the organization; or the protection of core competence for sharing, and otherwise should enter the shared knowledge base. (2) The integration of knowledge and the formation of common knowledge. This process helps us to establish cooperative sharing of knowledge base, mainly including three aspects: one is from all members of the organization after screening of knowledge; the second is from outside is conducive to the overall absorption and innovation of knowledge. Three is the basic knowledge and application knowledge of the new creation in the production and study cooperation. The sharing knowledge platform is based on the shared knowledge base, and all the operations are carried out in the range of shared knowledge base. (3) The absorption and application of knowledge. The process is mainly for the cooperation of the members of the knowledge retrieval service, facilitate the transfer of knowledge. At the same time, we can create a knowledge sharing network platform and portal services provide personalized interactive program cooperation interface and dynamic organization, in order to facilitate the exchange of knowledge, knowledge sharing which is the cooperation between organizations (mainly tacit knowledge) is the main way of knowledge; the search engine service can provide various types of search type to help the employees and organizations to quickly locate knowledge location; through the forum, virtual community, expert group discussion and other forms of organization to realize the sharing of synchronous and asynchronous interactive knowledge collaboration system based on portal services; evaluation of knowledge sharing service can make the organization more accurately positioning themselves in the knowledge sharing in the role.

Secondly, we make a simple analysis of some main modules. (1) Perception and action modules. Perception and action modules are related to the work of the subject, whose main role is to ensure the perception and function of the environment through the behavior of the main body. At the same time, the perception of the information will be based on the degree of urgency and complexity into different levels of processing. (2) Interaction module. The main purpose of the interactive module is to ensure the smooth communication between the main body, which is the basis for consultation, cooperation and coordination between the subjects. The main body of the interactive behavior is also a level; in general, the interaction module involves low layer network protocol, communication protocol, interaction protocol and high level interaction strategy and other information management. (3) Behavior reasoning module. Behavioral reasoning module is responsible for the behavior planning and decision-making of the nerve center, which consists of three levels, while closely related to the interaction module. The first layer (bottom) the reaction type structure of thought, in emergency or simple directly according to the rules of emergency behavior on the state of the environment to make real-time response; the second layer an environment or an interactive information and according to the agreed contract, protocol, planning or rules information, conventional behavior decision and management; the third layer is mainly responsible for the information cannot solve the first two layers, such as various types of contracts, protocol, planning, rule and strategy formulation and adjustment of the. It requires more intellectual support. So it is closely linked with the decision support environment. Decision support environment can be convenient to call the basic data module models and related knowledge and possible can provide corresponding man-machine interface makes the decision or domain experts can participate in the decision-making process. Behavior reasoning module can be used to achieve the basic structure of the main structure. (4) Knowledge module. The knowledge module is mainly used to ensure the relevant knowledge and data needed by the interactive module. This information was first obtained through the role of the planning ability, in the process of operation can be constantly revised.

Finally, we analyze the main body of the production of cooperative knowledge sharing cooperative interaction strategy. Three processes and four modules are introduced in the previous paper, and the basic framework of the interaction is given. In this framework, we can make a conscious dialogue between the subjects of knowledge sharing. But in general, this is only a fundamental guarantee; it does not involve specific issues in the background. In practical applications, the construction of multi 
agent system, or the purpose of the main interaction is always in order to achieve a certain goal or to solve a problem. Therefore, in order to solve the problem, we must adopt the corresponding interaction strategy for the specific problems of the knowledge sharing in the field of production. The choice of the interactive strategy is related to the planning ability of the subject role, such as the role of the individual knowledge base, the organization knowledge base and the related model, protocol, planning, rules and so on. In the process of interaction, there may be the role of resource dependence or conflict of interest, so the choice of interaction strategy is to solve these problems in the most appropriate way. Specifically, it is the choice of different interaction protocols for different interaction objects and different environment conditions.

\section{Summary}

In summary, subject between the collaborative interaction behaviors is production and study cooperation knowledge sharing important attribute and analysis of production and learning cooperation in collaborative interaction helps to help us promote the production of rational coordination, cooperation and consultation. Secondly, this paper classified according to the structure of the elements, analyzes the production and study cooperation knowledge sharing factors of the overall operation model, and the construction of the role played by the compound based on production cooperation study, knowledge sharing and cooperative interaction model, to illustrate the industry university cooperation and knowledge sharing process.

\section{References}

[1] Wu Sheng, SLin Cathy, Lin Tung- Ching .Exploring Knowledge Sharing in Virtual Team: A Social Exchange Theory Perspective. System Sciences, 2006, 1(1):26-36.

[2] Santoro M D, Chakrabarti A K. Firm size and technology centrality in industry-university interactions. Research Policy, 2002, 31: 1163-1180.

[3] Mary C Jones, Melinda Cline, Sherry Ryan. Exploring knowledge sharing in ERP implementation: an organizational culture framework. Decision Support Systems, 2006, 41(2): 411-434.

[4] Weiss L. Collection and connection: The anatomy of knowledge sharing in professional service. Organization Development Journal 1999, 17(4): 61-72.

[5] Grant, R. M. Prospering in dynamically-competitive environments: Organizational capability as knowledge integration. Organization Science, 1996, 7(4):375-388.

[6] Boer, M., Bosch, F. A. J. and Volberda, H. W. Management Organizational Knowledge Integration in the Emerging Multimedia Complex. Journal of Management Studies, 1999, 36(3): 379-398. 